\title{
Apoptosis Signaling Molecules as Treatment Targets in Head and Neck Squamous Cell Carcinoma
}

Thomas J. Ow, MD, MS ${ }^{1,2}$, Carlos Thomas ${ }^{2}$, Cory D. Fulcher, MD ${ }^{1}$, Jianhong Chen, PhD $^{3}$, Andrea López ${ }^{4}$, Denis E. Reyna, PhD ${ }^{4}$, Michael B. Prystowsky, MD, PhD ${ }^{2}$, Richard V. Smith, MD ${ }^{1,2,5}$, Bradley A. Schiff, MD1, Gregory Rosenblatt, PhD², Thomas J. Belbin, PhD ${ }^{2,8}$, Thomas M. Harris, $\mathrm{PhD}^{2}$, Geoffrey C. Childs, $\mathrm{PhD}^{2}$, Nicole Kawachi ${ }^{2}$, Nicolas F. Schlecht, $\mathrm{PhD}^{2,3,6,7}$, Evripidis Gavathiotis, PhD $^{4}$

1. Otorhinolaryngology-Head and Neck Surgery, Montefiore Medical Center/Albert Einstein College of Medicine, Bronx, NY, USA.

2.Pathology, Montefiore Medical Center/Albert Einstein College of Medicine, Bronx, NY, USA.

3.Cancer Prevention \& Control, Roswell Park Comprehensive Cancer Center, Buffalo, NY, USA.

4.Department of Biochemistry, Department of Medicine, Albert Einstein Cancer Center, Albert Einstein College of Medicine, Bronx, NY, USA

5.Surgery, Montefiore Medical Center/Albert Einstein College of Medicine, Bronx, NY

6.Epidemiology \& Population Health, Montefiore Medical Center/Albert Einstein College of Medicine, Bronx, NY, USA.

7. Medicine (Oncology), Montefiore Medical Center/Albert Einstein College of Medicine, Bronx, NY, USA.

8. Oncology, Memorial University of Newfoundland, Newfoundland and Labrador, Canada

\section{Abstract}

Objectives: To evaluate BCL-2 family signaling molecules in head and neck squamous cell carcinoma (HNSCC) and examine the ability of therapeutic agents with variable mechanisms of action to induce apoptosis in HNSCC cells.

Methods: mRNA expression of $B A K, B A X, B C L 2, B C L 2 L 1$, and $M C L 1$ were measured in the Cancer Genome Atlas (TCGA) head and neck cancer dataset, as well as in a dataset from a cohort at Montefiore Medical Center (MMC). Protein expression was similarly evaluated in a panel of HNSCC cell lines (HN30, HN31, HN5, MDA686LN, UMSCC47). Cell viability and Annexin V assays were used to assess the efficacy and apoptotic potential of a variety of agents (ABT-263 (navitoclax), A-1210477, and Bortezomib).

Corresponding Author: Thomas J. Ow, MD, MS, FACS, 3400 Bainbridge Ave, 3rd Fl MAP building, Bronx, NY 10467, Phone: 718-920-8488, Fax: 718-882-2463, thow@ montefiore.org.

Conflicts of Interest: none

Meeting Information:

Work from this manuscript was accepted for podium presentation at the $122^{\text {nd }}$ Annual Meeting of the Triological Society at COSM, Austin, Tx, May $3^{\text {rd }}, 2019$ 
Results: Expression of BAK, BAX, BCL2L1, and MCL1 were each significantly higher than expression of BCL2, in the TCGA and MMC datasets. Protein expression demonstrated the same pattern of expression when examined in HNSCC cell lines. Treatment with combined ABT-263 (navitoclax) / A-1210477, or with bortezomib demonstrated apoptosis responses that approached or exceeded treatment with staurospaurine control.

Conclusion: HNSCC cells rely on inhibition of apoptosis via BCL-xL and MCL-1 overexpression, and induction of apoptosis remains a potential therapeutic option, as long as strategies overcome redundant anti-apoptotic signals.

\section{Keywords}

Head and Neck Squamous Carcinoma; Apoptosis; ABT-263; A-1210477; Bortezomib

\section{Introduction}

Resisting cell death via evasion of apoptosis has been deemed a hallmark of cancer $^{1,2}$, and the survival of head and neck squamous cell cancer (HNSCC) depends on this hallmark. The upper aerodigestive tract squamous epithelium forms a stratified layer of cells that under normal circumstances terminally differentiate and die in an organized process that relies on several apoptosis signaling molecules ${ }^{3}$. It is not surprising that dysregulation of apoptosis is a critical component of the carcinogenesis process in $\mathrm{HNSCC}^{4}$. Indeed, TP53 is the most common mutation in $\mathrm{HNSCC}^{5-7}$, and a canonical model of $\mathrm{p} 53$ tumor suppression is to induce apoptosis in response to cell stressors such as DNA damage or activated oncogenes ${ }^{8}$.

Other genes involved in the intrinsic pathway of apoptosis, such as anti-apoptotic molecules $B C L 2, B C L 2 L 1$ (coding for the BCLxL protein), and $M C L 1$, as well as pro-apoptotic $B A X$ and $B A K$, appear to remain intact in $\mathrm{HNSCC}^{5}$. As one means to evade apoptosis, cancer cells commonly upregulate BCL-2 family pro-survival molecules ${ }^{9}$, and several studies have examined expression of these molecules and their role in treatment resistance in HNSCC $^{10-12}$. Recent work by our group has suggested that the pro-survival BCL-xL and MCL-1 proteins are frequently overexpressed in HNSCC, and we demonstrated that combined targeting of these two proteins resulted in synergistic effects on cell viability in vitro $^{13}$.

It appears that HNSCC depends on disruption of the normal apoptosis response, yet the intrinsic machinery remains functional. If true, activation of apoptosis could potentially be exploited in HNSCC, yielding an effective treatment strategy in HNSCC. In the current study, we confirm that BCL-xL and MCL-1 are expressed at high levels in HNSCC, along with pro-apoptotic BAX and BAK. Our previous publication demonstrated that inhibition of BCL-xL and MCL-1 resulted in synergistic effects on cell viability ${ }^{13}$. Here, we examine the apoptosis response using Annexin $\mathrm{V}$ assays after targeting HNSCC cells with several agents that alter apoptosis signaling - specifically treatment with the BCL2/BCL-xL inhibitor ABT-263 (navitoclax), the MCL-1 inhibitor A-1210477, and the proteasome inhibitor, Bortezomib. 


\section{Methods:}

\section{Gene Expression Microarray and RNAseq Data}

Data from a total of 496 HNSCC patients in The Cancer Genome Atlas (TCGA) cohort include level 3 RNA sequencing data (log2 transformed RSEM value, as reads per kilobase of transcript per million mapped reads) and clinical data (HPV status, tumor stage, and primary tumor site) downloaded from NCI Genomic Data Commons (GDC, https:// portal.gdc.cancer.gov/). Relative gene expression levels of targeted genes were presented as box-plots using GraphPad Prism ${ }^{\circledR}$ v. 8 (San Diego, CA). In parallel, the relative gene expression levels were examined from the targeted genes within tumor samples obtained and evaluated at Montefiore Medical Center (MMC). Ribonucleic acids (RNA) from HNSCC tumors were hybridization to the Illumina ${ }^{\circledR}$ HumanHT-12-v3 Expression BeadChip (Illumina ${ }^{\circledR}$, San Diego, CA) and data were normalized and evaluated, as described in previous reports ${ }^{13-15}$. For this analysis, evaluation was restricted to tumors from patients with histologically-proven HNSCC, without previous treatment, and without a history of a previous HNSCC primary tumor. Gene expression data from 131 HNSCC patients was $\log 2$ transformed, analyzed, and presented as box plots using GraphPad Prism ${ }^{\circledR}$ v. 8 (San Diego, CA).

Pairwise Pearson correlation analysis was conducted with relative gene expression levels of $B A X, B A K 1, B C L 2, B C L 211$, and MCL1 within TCGA and MMC cohorts using log2 transformed data. A correlation coefficient matrix was produced by combining pairwise correlation coefficients from TCGA and MMC data, respectively. The matrix was used for unsupervised hierarchical clustering using the Pheatmap package applied in R® statistical software.

\section{HNSCC cell culture}

Cell lines HN30, HN31, HN5, MDA686LN, and UMSCC47, were graciously provided by Dr. Jeffrey N. Myers, MD, PhD at the University of Texas, MD Anderson Cancer Center. Transfer agreements required the following permissions (HN30, HN31 - John Ensley, MD, Wayne State University; MDA686LN - Peter Sacks, MD, New York University/University of Texas MD Anderson Cancer Center; UMSCC47 - Thomas Carey, University of Michigan). All cells were grown in Dulbecco's Modified Eagle's Medium (DMEM) combined with $10 \%$ Fetal Bovine Serum (FBS), nonessential amino acids, sodium pyruvate and $1 \%$ antibiotic - penicillin/strepotmycin. Incubation was maintained at $37^{\circ} \mathrm{C}, 5 \% \mathrm{CO} 2$. Stocks of each line used were authenticated with short tandem repeat (STR) genotyping.

\section{Western blot analysis}

Whole cell lysates from HSNCC cells were prepared in radioimmunoprecipitation assay (RIPA) buffer containing protease inhibitor cocktail (Pierce ${ }^{\mathrm{TM}}$, Thermo Scientific, Rockford, IL, USA). Protein concentrations were measured using $\mathrm{DC}^{\mathrm{TM}}$ protein assay (Bio-Rad ${ }^{\mathrm{TM}}$, Hercules, CA, USA). Sodium dodecyl sulfate-polyacrilamide gel electrophoresis (SDSPAGE) was run at $80 \mathrm{~V}$ and membrane immunoblotted overnight. Primary antibodies were incubated in $1 \%$ bovine serum albumin (BSA)/Tris-buffered saline with $0.1 \%$ Tween-20 at concentrations varying from 1:500 - 1:5000 (anti- $\beta$-actin - 8H10D10,1:5000; anti-BCL-2 
(124) 15071S, 1:500; anti-mcl-1 (D35A5) 5453S; anti-bax 2772S, 1:500; anti-BCL-xL (54H6) 2764s, Cell Signaling, 1:500; each from Cell Signaling, Danvers, MA, USA; and anti-BAK,1:500,NT, EMD Millipore Corp., Billenca, MA, USA. Fluorescent secondary antibodies against mouse or rabbit were sourced from LI-COR ${ }^{\mathrm{TM}}$ (Lincoln, NE, USA). Immunolabeled proteins were visualized with the LI-COR® Odyssey® FC Imaging System. Protein quantification was determined from 3 independent experiments using image studio software (LI-COR ${ }^{\circledR}$ Biosciences, Lincoln, NE).

\section{Cell Viability Assays - Screening}

A pipetting robot was utilized to plate cells and test agents in a rapid, high throughput fashion. HNSCC cells (1500 cells/well) were seeded in 96-well white plates. Cells were incubated with serial dilutions of Bortezomib. Control (untreated) wells were maintained in parallel dilutions of dissolvent DMSO. Cell Titer-Glo (Promega, Madison, WI), was used to assess cell viability according to the manufacturer's protocol. The TECAN M200 microplate reader was used to measure luminescence. Viability assays were performed in duplicates and the data normalized to vehicle-treated control wells. IC50 values were determined by nonlinear regression analysis using Prism software (GraphPad Software, La Jolla, CA).

3-(4,5-dimethylthiazol-2-yl)-2,5-diphenyltetrazolium bromide (MTT) Cell Viability Assay HNSCC cell lines were plated on 96-well plates at a density of 1,500 cells/well. Cells were allowed to incubate for 24 hours before addition of bortezomib, with doses ranging from

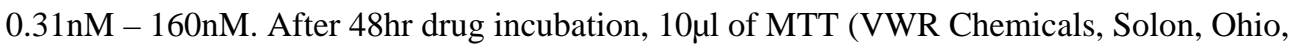
USA) at $5 \mathrm{mg} / \mathrm{ml}$ was added until visualization of formazan crystals by 150 minutes. Cell viability was determined by spectrophotometry at 570nM. IC50 values were calculated by non-linear regression analysis of the recorded absorbances with respect to vehicle control wells using GraphPad Prism ${ }^{\circledR}$ v. 8 (San Diego, CA).

\section{Bortezomib treatment of HNSCC}

HNSCC cells were plated onto $10 \mathrm{~cm}$ plates and allowed to grow for $24 \mathrm{~h}$. The following day, DMEM containing 5nM or 10nM Bortezomib (EMD Millipoe Corp, USA) was added and incubated for $24 \mathrm{~h}$ at $37^{\circ} \mathrm{C}$ with $5 \% \mathrm{CO}$. Whole cell lysate was extracted in RIPA buffer at the end of incubation.

\section{Annexin V Apoptosis Assay}

125,000 HNSCC cells were seeded in each well of 6 well plates and incubated for 24 hours. After 24hrs, HNSCC were treated with apoptosis inducing agents at the following concentrations: $1 \mu \mathrm{M}$ Staurosporine (Adipogen Life Sciences, San Diego, CA), $5 \mu \mathrm{M}$

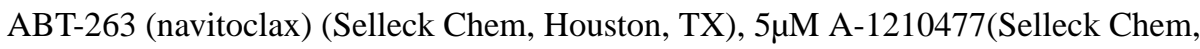
Houston, TX), and 5 $\mu \mathrm{M}$ ABT-263 (navitoclax)/5 $\mu \mathrm{M}$ A-1210477 combination 5nM Bortezomib, and 10nM Bortezomib. Cells were incubated for another 48hours and harvested with accutase, washed in PBS and stained with PE Annexin V Apoptosis Detection Kit I (BD Biosciences ,San Diego, CA). Analysis was conducted by flow analyzer (BD LSRII Yellow). Flow cytometer data was analyzed and figures produced by Flow ${ }^{\mathrm{TM}}{ }^{\mathrm{v} 10}$ software (FlowJo, LLC, Ashland, OR). 


\section{Statistical Analyses}

Mean averages were calculated for each transcript assessed from gene expression data in TCGA and MMC datasets, and transcripts were compared against each other using twotailed Student T-tests. Median or mean averages were calculated where appropriate for Annexin V data and for protein quantification, and box-plots were created to represent data, where described. For statistical analyses, a p-value of 0.05 was accepted as the threshold for significance. Statistical analyses for specific assays are included in more detail in individual sections above.

\section{Results}

\section{Evaluation of Expression of BCL-2 Family Transcripts in TCGA and MMC HNSCC}

The relative gene expression of BCL2, BCL2L1, MCL1, BAX, and BAK1 were evaluated in TCGA dataset (Figure 1A) and the MMC dataset (Figure 1B). Both datasets demonstrated a similar pattern of expression, and expression of pro-apoptotic $B A X$ and $B A K 1$, as well as anti-apoptotic $B C L 2 L 1$ and $M C L 1$ were all significantly higher than expression of $B C L 2$ based on comparisons with Student T-tests in both datasets. Correlation matrices for these transcripts were generated for TCGA (Figure 1C) and MMC data (Figure 1D) in an attempt to examine the balance of pro- and anti-apoptotic transcripts. In both TCGA and MMC datasets the only strong anti-correlation was noted between $B C L 2$ and $B A K 1$.

\section{Expression of BCL Family Proteins in HNSCC Cell Lines}

HNSCC cell lines HN30, HN31, HN5, MDA686LN, and UMSCC47 were used for further studies. These lines represent HNSCC cells from a variety of different HNSCC sites (Table 1), and included a cell line known to carry wildtype TP53 (HN30), its isogenic line derived from a lymph node metastasis harboring mutant copies of TP53 (HN31), as well as an HPVpositve cell line (UMSCC47) ${ }^{16}$. Baseline protein expression of BCL-2, BCL-xL, MCL1, $\mathrm{BAX}$, and BAK were evaluated (Figure 2A) and average values after quantification of these proteins from multiple blots demonstrated a pattern of expression similar to gene transcription seen in the patient cohorts, with low BCL2 expression, and relatively higher expression of BCL-xL, MCL1, BAX, and BAK (Figure 2B).

\section{Apoptosis Response After Targeting BCL-xL and MCL1}

Since it was apparent that HNSCC expresses both pro- and anti-apoptotic signaling molecules, several approaches of targeting apoptosis were evaluated. Data presented above suggests that multiple redundant mechanisms are in place for HNSCC to resist apoptosis. For this reason, strategies that alter multiple anti-apoptosis mechanisms were chosen for further evaluation. Our previous work demonstrated that concurrently targeting BCL2, BCL$\mathrm{xL}$ and MCL-1 resulted in synergistic reduction in cell viability in multiple HNSCC cell lines ${ }^{13}$. In the current study, apoptosis was evaluated in the cell line panel evaluating Annexin V after treatment with ABT-263 (Navitoclax), after treatment with the MCL-1 inhibitor, A-1210477, and after combination treatment. Combination treatment significantly increased apoptosis compared to single-agent treatment in all cell lines, and reached levels 
near or greater than samples treated with the control apoptosis agent staurosporine (Figure $3)$.

\section{Cell Viability and Apoptosis Response After Bortezomib Treatment}

Bortezomib has previously been shown to induce apoptosis via several mechanisms ${ }^{17}$, such as inducing the unfolded protein response $\mathrm{e}^{18}$, and via alterations in the levels and activity of apoptosis signaling molecules ${ }^{19,20}$. Therefore, the cell viability and apoptosis response to bortezomib was evaluated in our HNSCC cell line panel. Bortezomib showed exquisite responses on drug screening (supplemental Figure 1), and responses were validated using MTT assays. Results demonstrated IC50 doses ranging from $1.6-10.15 \mathrm{nM}$, confirming that HNSCC cells are highly sensitive to Bortezomib (supplemental Table 1, and Figure 4). When apoptosis responses were evaluated with Annexin V assays, HNSCC cell lines each demonstrated a dose-dependent response to Bortezomib, with responses nearing or exceeding staurosporine controls at 10nM of Bortezomib in HN30, HN31, and HN5 (Figure 5). Protein levels of BCL2, BCL-xL, MCL1, BAX, and BAK were evaluated by western blot before and after Bortezomib treatment, however there were no significant or consistent changes noted in the overall protein expression of these factors (Supplemental Figure 2).

\section{Discussion}

In this report, we provide evidence that BCL-xL and MCL-1 play an important role in the mechanism by which HNSCC tumors resist apoptosis. We also demonstrate that activation of apoptosis remains available as a treatment strategy in HNSCC as long as one can overcome redundant mechanisms that keep apoptosis in check in this disease. Anti-apoptotic BCL2L1 and MCL1 transcripts were highly expressed in the TCGA dataset, as well as in an archival dataset from our institution. These same proteins were elevated in HNSCC cell lines. Pro-apoptotic BAX and BAK were also expressed in patient samples and HNSCC cell lines. This expression pattern suggests that targeting apoptosis via inhibition of both BCL$\mathrm{xL}$ and MCL1 would be effective and potentially synergistic, which is what was observed in HNSCC lines in our recent publication ${ }^{13}$. In this current follow-up report, we show that combination ABT-263 (navitoclax) plus A-1210477, collectively inhibiting BCL-xL, BCL2, and MCL1, results in a strong apoptosis response. The redundancy in anti-apoptotic signaling also provides some rationale for the efficacy of bortezomib, which potentially activates apoptosis via several pathways as sequelae after proteasome inhibition.

BCL2 family proteins have been examined previously in HNSCC. Work by Michaud and colleagues suggests that BCL2 over-expression is associated with treatment failure and cisplatin resistance in HNSCC cell lines, whereas BCL-xL expression showed no such correlation $^{11}$. Alternatively, Bauer and colleagues examined BCL-xL expression among the Department of Veteran's Affairs Laryngeal Cancer Study, and found that high BCL-xL expression was associated with a low rate of laryngeal preservation ${ }^{10}$. Whether these markers are truly prognostic remains an open question, and our study did not focus on the utility of BCL2 family proteins as prognostic biomarkers. Our data suggest that high expression of BCL-xL is perhaps a hallmark of HNSCC in general, and thus may not be a good prognostic marker. However, BCL-xL may certainly be a valid treatment target. We 
also demonstrate that targeting BCL-xL is much more effective when combined with MCL-1 inhibition, resulting in substantially increased apoptosis, supporting cell viability results from our previous study ${ }^{13}$.

Targeting BCL-2 family proteins has been an area of research in HNSCC, and as newer agents targeting apoptosis are developed, exploration in this field has expanded. $\mathrm{Li}$ and colleagues published proof-of-concept work that demonstrated efficacy of targeting BCL2 and BCL-xL using cell permeable peptides derived from the BH3 domains of Bad, Bak, and $\mathrm{Bax}^{21}$. The in vitro efficacy of the first-generation BCL2/BCL-xL inhibitor, ABT-737, has been examined in HNSCC cells in combination with cisplatin and etoposide ${ }^{22}$, and also with sorafenib ${ }^{23}$. Other apoptosis-acting therapeutics, including AT- $101^{24}$, obatoclax ${ }^{25}$, and venetoclax ${ }^{26}$, have been studied as single agents. Several studies, prior to the advent of newer MCL1-directed molecular agents, have examined down-regulation of MCL1 as an effective mechanism of action of several drugs in HNSCC. Early in vitro work studying HNSCC work showed that inhibition of MCL1 using antisense oligonucleotides could enhance the efficacy of several chemotherapeutic agents ${ }^{27}$. Zhang et al. studied the proteasome inhibitor carfilzomib in HNSCC and found that upregulation of MCL1 led to resistance to this therapy, while inhibition of MCL-1 with si-RNA resulted in improved responses ${ }^{28}$. The epidermal growth factor receptor (EGFR) inhibitor, afatinib, has also been shown to down-regulate MCL-1 and induce apoptosis in $\mathrm{HNSCC}^{29}$. These studies support the importance of MCL-1 in inhibiting apoptosis and resisting standard treatments in HNSCC.

MCL-1-specific inhibitors have been developed recently, and we are the first to our knowledge to report activity of A-1210477 in HNSCC, both in a recent report ${ }^{13}$ and this current study. We demonstrate that combining MCL-1 inhibition with BCL-2/BCL-xL/BCLw inhibition via ABT-263 (navitoclax) results in a substantial increase in apoptosis across multiple HNSCC cell lines. Though targeting of specific BCL-family molecules may be efficacious in certain malignancies, such as targeting BCL-2 with venetoclax in chronic lymphocytic leukemia ${ }^{30}$, it seems that combination therapy targeting multiple BCL-family proteins may be necessary in most solid tumors. Inoue-Yamauchi and colleagues recently demonstrated that indirect downregulation of MCL-1 with either anthracyclines or CDK9 inhibition was necessary to overcome resistance to ABT-263 in small cell lung cancer cells $^{31}$. In 2015, Maji et al. demonstrated that MCL-1 knockdown improved response of oral cavity squamous cell cancer cells to the BCL-2/BCL-xL inhibitor ABT-737, and they showed that the pan- anti-apoptotic inhibitor sabutoclax was effective in a murine model as a single agent ${ }^{32}$. Another recent study demonstrated that mantel cell lymphomas that resist treatment with BCL-2 specific venetoclax can be overcome with the synthetically lethal combination of venetoclax and the MCL-1 inhibitor S63845 33 .

In this study, we describe bortezomib as an agent that induces apoptosis in HNSCC. As stated previously, bortezomib activates apoptosis via the unfolded protein response and ER stress following proteasome inhibition ${ }^{18,34}$. Additionally, bortezomib may alter the balance of BCL2 family apoptosis signaling molecules, several of which are regulated by proteosomal degradation ${ }^{17}$. Bortezomib has received significant attention in HNSCC. Li and colleagues demonstrated that bortezomib could activate apoptosis and synergize with 
cisplatin in $\mathrm{HNSCC}^{20}$. Other studies have suggested that bortezomib can liberate p53, contributing to apoptosis in HPV-positive $\mathrm{HNSCC}^{20,35}$. Chen and colleagues were among the first to examine bortezomib in HNSCC cells, and also demonstrated in vitro activity at nanomolar ranges ${ }^{36}$. Unfortunately, promising preclinical data have not translated into clinical activity using bortezomib. Bortezomib was examined alone and in combination with irinotecan in HNSCC patients in a large, multi- institutional phase-II trial supported by the Eastern Cooperative Group (ECOG E1304), with discouraging results ${ }^{37}$. Though bortezomib is approved for the treatment of multiple myeloma, the drug has not found utility in solid tumors. It is unclear whether this is due to limited activity of the drug, the bioavailability and pharmacokinetics of the agent ${ }^{38}$, or inherent resistance factors within tumors. Nevertheless, our study showed that bortezomib could induce apoptosis in HNSCC at very low doses, and we speculate that this may be secondary to the drug's ability to overcome several redundant apoptosis resistance mechanisms in HNSCC.

\section{Conclusions}

In summary, though evasion of apoptosis is necessary for cancer cell survival, HNSCC cells maintain intact apoptosis machinery, and appear to rely on inhibition of apoptosis via BCL$\mathrm{xL}$ and MCL-1 over-expression. Therefore, induction of apoptosis remains a potential therapeutic option, as long as strategies overcome redundant anti-apoptotic signals inherent to HNSCC cancer cell survival. Our work studying the activity of ABT-263, A-1210477, and bortezomib, support this concept.

\section{Supplementary Material}

Refer to Web version on PubMed Central for supplementary material.

\section{Funding:}

Thomas J. Ow's contribution was supported in part by the Triological Society Career Development Award, NIHNCI grant 2K12 CA132783-06, and NIH-NIDCR grant 1 K23 DE027425-01. E.G. and D.E.R. were supported from NIH-NCI grant CA178394 and an award from the Pershing Square Sohn Cancer Research Alliance. AL was supported from a T32 Training grant T32 AG 23475. The authors also acknowledge support from the Albert Einstein Cancer Center (NIH-NCI P30CA013330), and specifically the flow cytometry core facility, part of the Albert Einstein shared resources, as well as Roswell Park Comprehensive Cancer Center (CA016056). The manuscript content is solely the responsibility of the authors and do not necessarily represent the official views of the National Institute of Health (NIH).

\section{References}

1. Hanahan D, Weinberg RA. The hallmarks of cancer. Cell. 2000;100(1):57-70. [PubMed: 10647931]

2. Hanahan D, Weinberg RA. Hallmarks of cancer: the next generation. Cell. 2011;144(5):646-674. [PubMed: 21376230]

3. Lippens S, Denecker G, Ovaere P, Vandenabeele P, Declercq W. Death penalty for keratinocytes: apoptosis versus cornification. Cell death and differentiation. 2005;12 Suppl 2:1497-1508. [PubMed: 16247497]

4. Michcik A, Cichorek M, Daca A, et al. Tobacco smoking alters the number of oral epithelial cells with apoptotic features. Folia histochemica et cytobiologica. 2014;52(1):60-68. [PubMed: 24802962]

5. Comprehensive genomic characterization of head and neck squamous cell carcinomas. Nature. 2015;517(7536):576-582. [PubMed: 25631445] 
6. Agrawal N, Frederick MJ, Pickering CR, et al. Exome sequencing of head and neck squamous cell carcinoma reveals inactivating mutations in NOTCH1. Science (New York, NY). 2011;333(6046):1154-1157.

7. Stransky N, Egloff AM, Tward AD, et al. The mutational landscape of head and neck squamous cell carcinoma. Science (New York, NY). 2011;333(6046):1157-1160.

8. Goh AM, Coffill CR, Lane DP. The role of mutant p53 in human cancer. The Journal of pathology. 2011;223(2):116-126. [PubMed: 21125670]

9. Yip KW, Reed JC. Bcl-2 family proteins and cancer. Oncogene. 2008;27(50):6398-6406. [PubMed: 18955968]

10. Bauer JA, Kumar B, Cordell KG, et al. Targeting apoptosis to overcome cisplatin resistance: a translational study in head and neck cancer. International journal of radiation oncology, biology, physics. 2007;69(2 Suppl):S106-108.

11. Michaud WA, Nichols AC, Mroz EA, et al. Bcl-2 blocks cisplatin-induced apoptosis and predicts poor outcome following chemoradiation treatment in advanced oropharyngeal squamous cell carcinoma. Clinical cancer research : an official journal of the American Association for Cancer Research. 2009;15(5):1645-1654. [PubMed: 19240170]

12. Nichols AC, Finkelstein DM, Faquin WC, et al. Bcl2 and human papilloma virus 16 as predictors of outcome following concurrent chemoradiation for advanced oropharyngeal cancer. Clinical cancer research : an official journal of the American Association for Cancer Research. 2010;16(7):2138-2146. [PubMed: 20233885]

13. Ow TJ, Fulcher CD, Thomas C, et al. Optimal targeting of BCL-family proteins in head and neck squamous cell carcinoma requires inhibition of both BCL-xL and MCL-1. Oncotarget. 2019;10(4):494-510. [PubMed: 30728900]

14. Belbin TJ, Bergman A, Brandwein-Gensler M, et al. Head and neck cancer: reduce and integrate for optimal outcome. Cytogenetic and genome research. 2007;118(2-4):92-109. [PubMed: 18000360]

15. Belbin TJ, Schlecht NF, Smith RV, et al. Site-specific molecular signatures predict aggressive disease in HNSCC. Head and neck pathology. 2008;2(4):243-256. [PubMed: 20614290]

16. Sano D, Xie TX, Ow TJ, et al. Disruptive TP53 mutation is associated with aggressive disease characteristics in an orthotopic murine model of oral tongue cancer. Clinical cancer research : an official journal of the American Association for Cancer Research. 2011;17(21):6658-6670. [PubMed: 21903770]

17. Mujtaba T, Dou QP. Advances in the understanding of mechanisms and therapeutic use of bortezomib. Discovery medicine. 2011;12(67):471-480. [PubMed: 22204764]

18. Obeng EA, Carlson LM, Gutman DM, Harrington WJ Jr., Lee KP, Boise LH. Proteasome inhibitors induce a terminal unfolded protein response in multiple myeloma cells. Blood. 2006;107(12):4907-4916. [PubMed: 16507771]

19. Nikiforov MA, Riblett M, Tang WH, et al. Tumor cell-selective regulation of NOXA by c-MYC in response to proteasome inhibition. Proceedings of the National Academy of Sciences of the United States of America. 2007;104(49):19488-19493. [PubMed: 18042711]

20. Li C, Li R, Grandis JR, Johnson DE. Bortezomib induces apoptosis via Bim and Bik up-regulation and synergizes with cisplatin in the killing of head and neck squamous cell carcinoma cells. Molecular cancer therapeutics. 2008;7(6):1647-1655. [PubMed: 18566236]

21. Li R, Boehm AL, Miranda MB, Shangary S, Grandis JR, Johnson DE. Targeting antiapoptotic Bcl-2 family members with cell-permeable $\mathrm{BH} 3$ peptides induces apoptosis signaling and death in head and neck squamous cell carcinoma cells. Neoplasia (New York, NY). 2007;9(10):801-811.

22. Li R, Zang Y, Li C, Patel NS, Grandis JR, Johnson DE. ABT-737 synergizes with chemotherapy to kill head and neck squamous cell carcinoma cells via a Noxa-mediated pathway. Molecular pharmacology. 2009;75(5):1231-1239. [PubMed: 19246337]

23. Kim LH, Shin JA, Jang B, et al. Sorafenib potentiates ABT-737-induced apoptosis in human oral cancer cells. Archives of oral biology. 2017;73:1-6. [PubMed: 27632413]

24. Zerp SF, Stoter TR, Hoebers FJ, et al. Targeting anti-apoptotic Bcl-2 by AT-101 to increase radiation efficacy: data from in vitro and clinical pharmacokinetic studies in head and neck cancer. Radiation oncology (London, England). 2015;10:158. 
25. Yazbeck VY, Li C, Grandis JR, Zang Y, Johnson DE. Single-agent obatoclax (GX15-070) potently induces apoptosis and pro-survival autophagy in head and neck squamous cell carcinoma cells. Oral oncology. 2014;50(2):120-127. [PubMed: 24216166]

26. Wang Y, Wang Y, Fan X, et al. ABT-199-mediated inhibition of Bcl-2 as a potential therapeutic strategy for nasopharyngeal carcinoma. Biochemical and biophysical research communications. 2018;503(3):1214-1220. [PubMed: 30017199]

27. Skoda C, Erovic BM, Wachek V, et al. Down-regulation of Mcl-1 with antisense technology alters the effect of various cytotoxic agents used in treatment of squamous cell carcinoma of the head and neck. Oncology reports. 2008;19(6):1499-1503. [PubMed: 18497956]

28. Zang Y, Thomas SM, Chan ET, et al. Carfilzomib and ONX 0912 inhibit cell survival and tumor growth of head and neck cancer and their activities are enhanced by suppression of Mcl-1 or autophagy. Clinical cancer research : an official journal of the American Association for Cancer Research. 2012;18(20):5639-5649. [PubMed: 22929803]

29. Liu X, Lv Z, Zou J, et al. Afatinib down-regulates MCL-1 expression through the PERKeIF2alpha-ATF4 axis and leads to apoptosis in head and neck squamous cell carcinoma. American journal of cancer research. 2016;6(8):1708-1719. [PubMed: 27648360]

30. Roberts AW, Davids MS, Pagel JM, et al. Targeting BCL2 with Venetoclax in Relapsed Chronic Lymphocytic Leukemia. The New England journal of medicine. 2016;374(4):311-322. [PubMed: 26639348]

31. Inoue-Yamauchi A, Jeng PS, Kim K, et al. Targeting the differential addiction to anti-apoptotic BCL-2 family for cancer therapy. Nature communications. 2017;8:16078.

32. Maji S, Samal SK, Pattanaik L, et al. Mcl-1 is an important therapeutic target for oral squamous cell carcinomas. Oncotarget. 2015;6(18):16623-16637. [PubMed: 26009874]

33. Prukova D, Andera L, Nahacka Z, et al. Co-targeting of BCL2 with venetoclax and MCL1 with S63845 is synthetically lethal in vivo in relapsed mantle cell lymphoma. Clinical cancer research : an official journal of the American Association for Cancer Research. 2019.

34. Nawrocki ST, Carew JS, Dunner K Jr., et al. Bortezomib inhibits PKR-like endoplasmic reticulum (ER) kinase and induces apoptosis via ER stress in human pancreatic cancer cells. Cancer research. 2005;65(24):11510-11519. [PubMed: 16357160]

35. Seltzsam S, Ziemann F, Dreffke K, et al. In HPV-Positive HNSCC Cells, Functional Restoration of the p53/p21 Pathway by Proteasome Inhibitor Bortezomib Does Not Affect Radio- or Chemosensitivity. Translational oncology. 2019;12(3):417-425. [PubMed: 30554133]

36. Chen Z, Ricker JL, Malhotra PS, et al. Differential bortezomib sensitivity in head and neck cancer lines corresponds to proteasome, nuclear factor-kappaB and activator protein-1 related mechanisms. Molecular cancer therapeutics. 2008;7(7):1949-1960. [PubMed: 18645005]

37. Gilbert J, Lee JW, Argiris A, et al. Phase II 2-arm trial of the proteasome inhibitor, PS-341 (bortezomib) in combination with irinotecan or PS-341 alone followed by the addition of irinotecan at time of progression in patients with locally recurrent or metastatic squamous cell carcinoma of the head and neck (E1304): a trial of the Eastern Cooperative Oncology Group. Head \& neck. 2013;35(7):942-948. [PubMed: 22791234]

38. Tan CRC, Abdul-Majeed S, Cael B, Barta SK. Clinical Pharmacokinetics and Pharmacodynamics of Bortezomib. Clinical pharmacokinetics. 2019;58(2):157-168. [PubMed: 29802543] 
A.

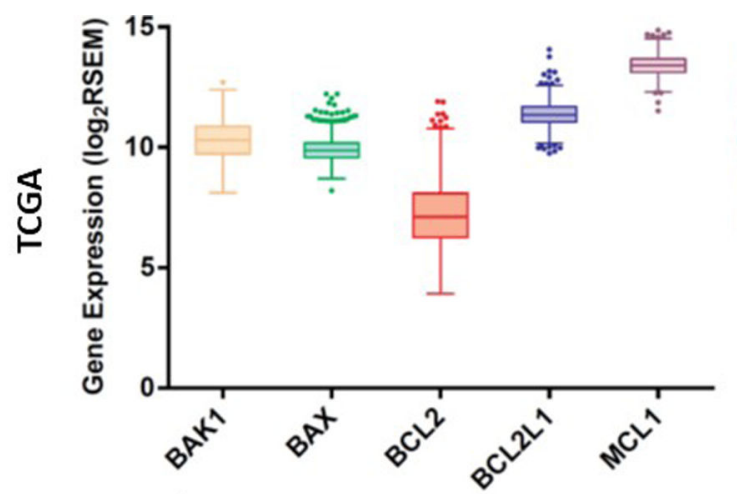

B.

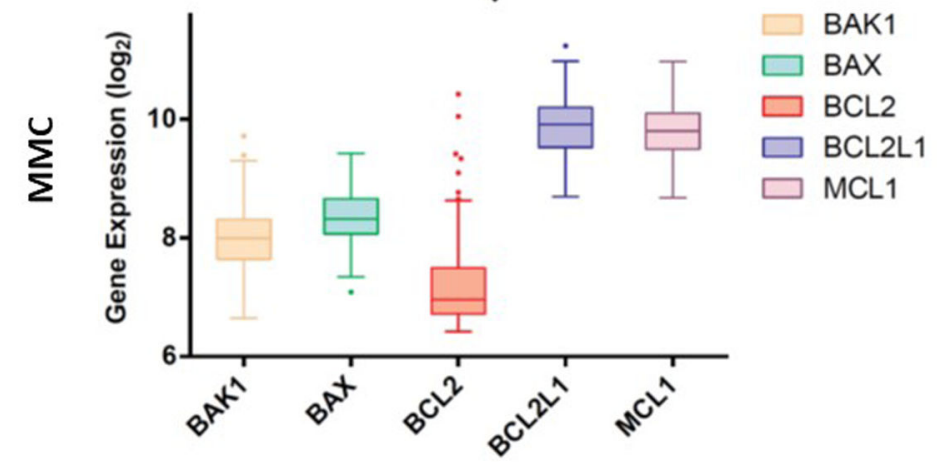

C. TCGA

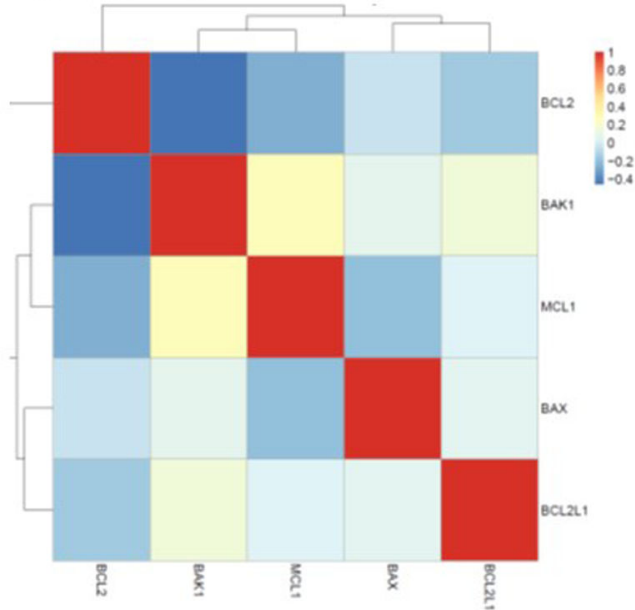

D. $\mathrm{MMC}$

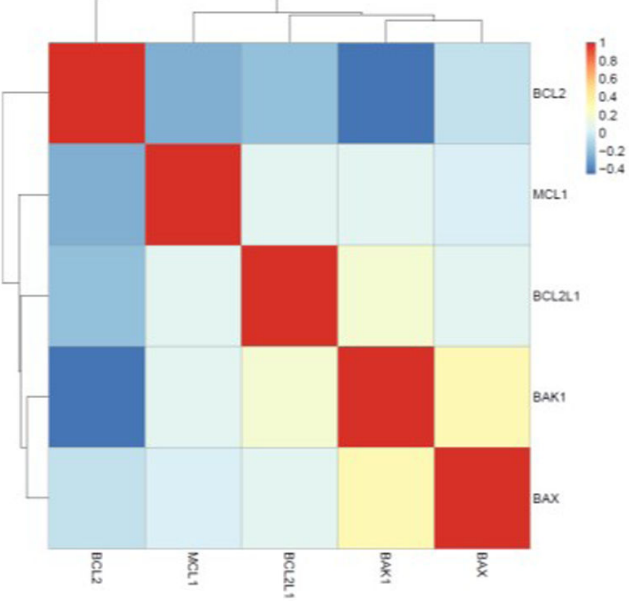

Figure 1.

Relative gene expression levels of $B a x$ (green), Bak1 (salmon), Bcl2 (red), Bcl2l1 (blue), and Mcl1 (purple) of the HNSCC cases within TCGA (A) and MMC (B) cohorts, respectively. The horizontal middle line is the median; the height of the boxes indicate the interquartile range (IQR) with top and bottom marking the 25 and 75 percentiles, respectively; dots mark outliers. The bottom panel heatmap shows pairwise correlation coefficients of relative gene expression levels of Bax, Bak1, Bcl2, Bcl211, and Mcl1 of the HNSCC cases within TCGA (C) and MMC (D) cohorts, respectively. Red denotes a positive correlation and blue denotes a negative correlation. 


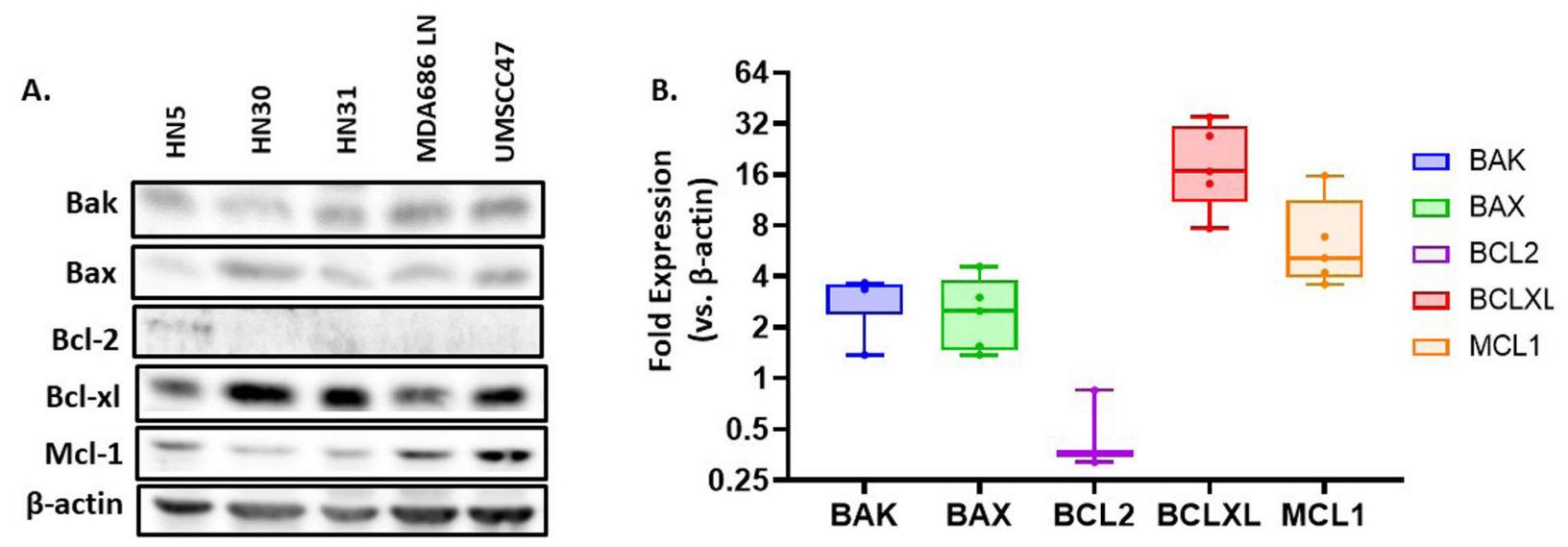

Figure 2.

Representative western blot data demonstrating protein expression of $B A X, B A K, B C L 2$, $B C L-x L$, and $M C L-1$ in HNSCC cell lines (HN5, HN30, HN31, MDA686LN, and UMSCC47) (A). Quantification of protein bands normalized to $\beta$-Actin loading controls based on replicated data for each cell line, and pooled across all lines examined. The horizontal middle line is the median fold expression; the height of the box indicates the interquartile range (IQR) (B). 
A.

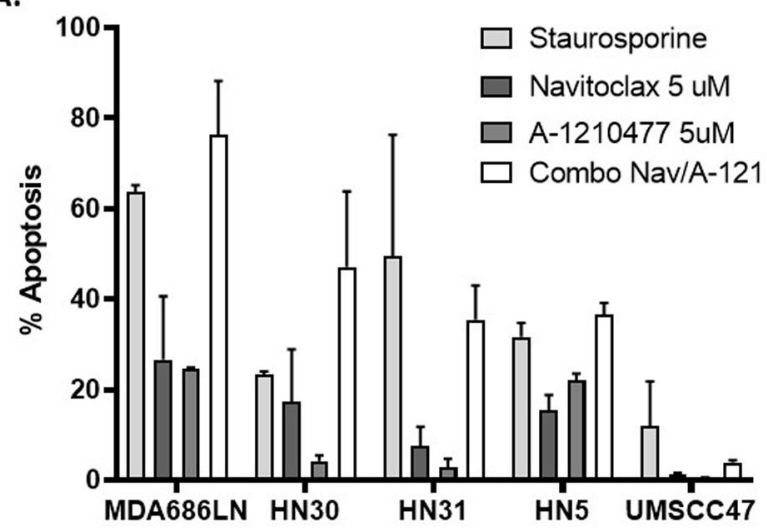

B.

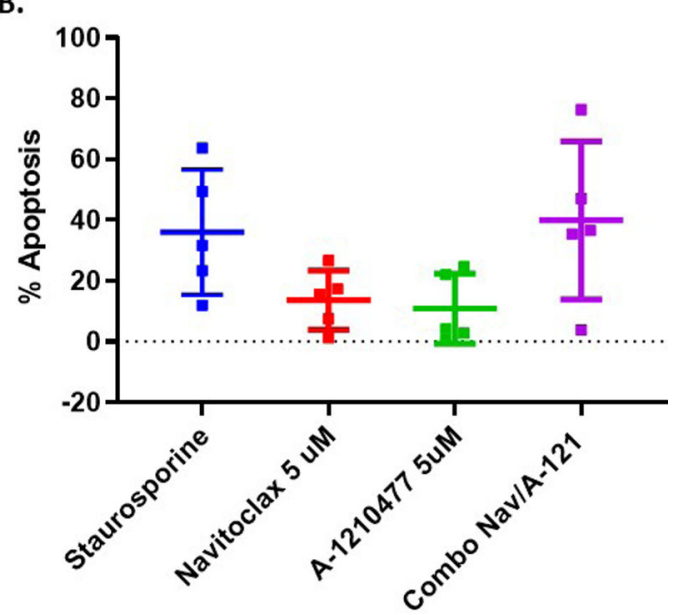

Figure 3.

Apoptosis response in HNSCC cell lines based on Annexin V assays after treatment with staurosporine as a control showing, ABT-263 (navitoclax), A-1210477, and combination ABT-263 (navitoclax)/A-1210477. Data are presented for each cell line (A.), and pooled (B.), where the horizontal line represents the mean value for all assays and the whiskers represent the standard deviation. 
A.

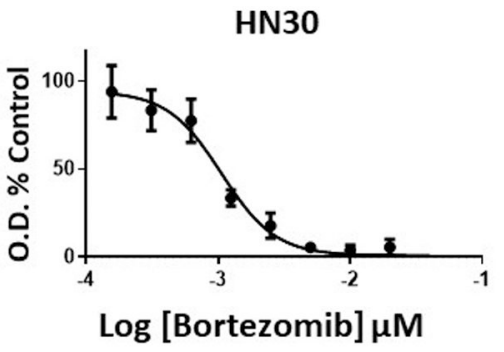

D.

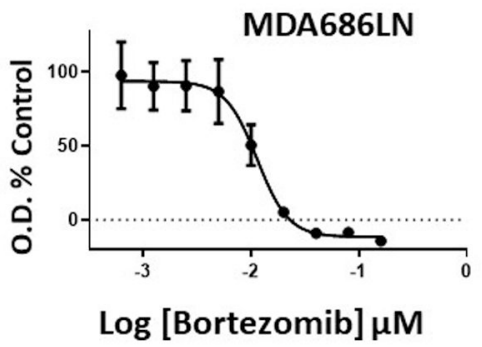

B.

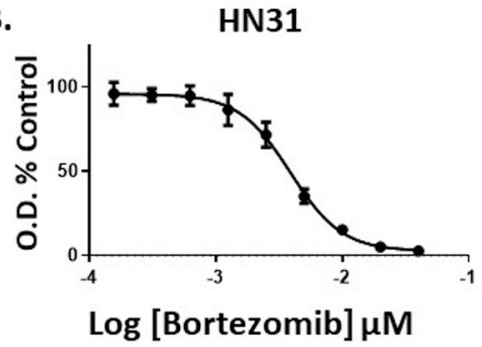

E.

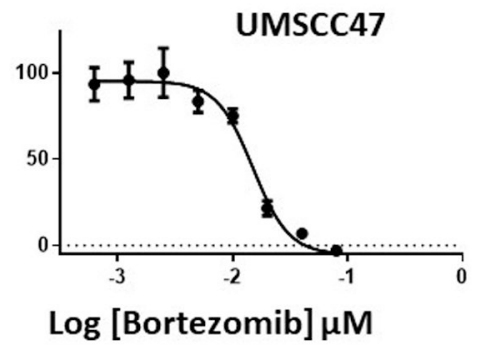

C. HN5

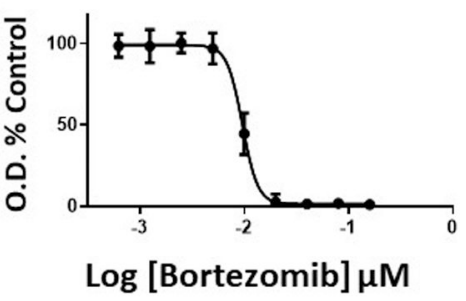

Figure 4.

Representative dose-response curves after bortezomib treatment for HNSCC cell lines HN30 (A), HN31 (B), HN5 (C), MDA686LN (D), and UMSCC47 (E). 
A.

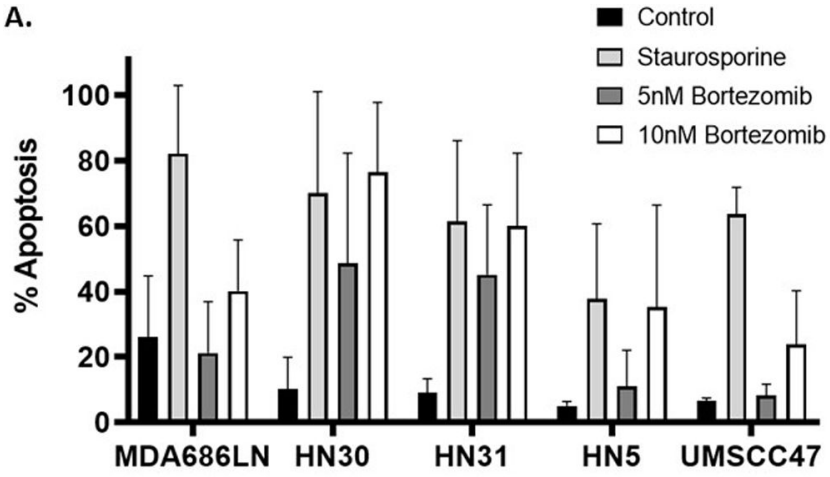

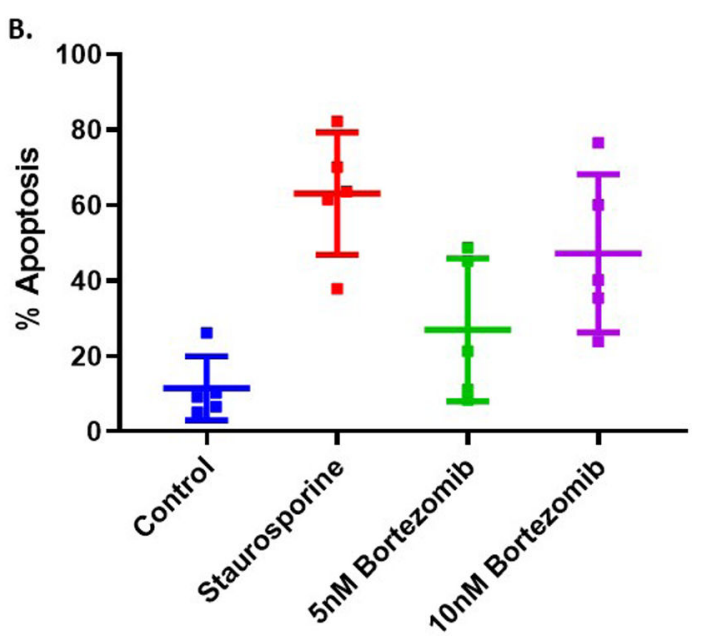

Figure 5.

Apoptosis response in HNSCC cell lines based on Annexin V assays after treatment with staurosporine as a positive control, and Bortezomib at 5nM and $10 \mathrm{nM}$. Data are presented for each cell line (A), and pooled (B), where the horizontal line represents the mean value for all assays and the whiskers represent the standard deviation. 
Table 1.

Head and Neck Cancer Cell Line Characteristics

\begin{tabular}{lll}
\hline Cell Line & Site & HPV Status \\
HN30* & Hypopharynx & Negative \\
HN31* & Lymph node metastasis & Negative \\
HN5 & Oral Cavity & Negative \\
MDA686LN & Lymph node metastasis (Oropharynx) & Negative \\
UMSCC47 & Oral Cavity & Positive \\
\hline$*$ &
\end{tabular}


Table 2.

IC50 values for Bortezomib in HNSCC cell lines

\begin{tabular}{lcc}
\hline Cell line & IC50 (nM) & SD $(\mathbf{n M})$ \\
HN30 & 1.62 & \pm 0.74 \\
HN31 & 3.45 & \pm 0.64 \\
HN 5 & 10 & \pm 0.57 \\
MDA686LN & 10.15 & \pm 1.63 \\
UMSCC47 & 10.15 & \pm 6.72 \\
\hline
\end{tabular}

$\mathrm{SD}=$ standard deviation

$\mathrm{nM}=$ Nanomolar

HNSCC $=$ Head and neck squamous cell carcinoma 